\title{
Coexpression of hyperactivated AKT1 with additional genes activated in leukemia drives hematopoietic progenitor cells to cell cycle block and apoptosis
}

Yan-juan Tang, Camilla Halvarsson, Amanda Nordigården, Komal Kumar, Josefine Åhsberg, Emma Rörby, Wan Man Wong and Jan-Ingvar Jönsson

\section{Linköping University Post Print}

\section{Tweet}

N.B.: When citing this work, cite the original article.

Original Publication:

Yan-juan Tang, Camilla Halvarsson, Amanda Nordigården, Komal Kumar, Josefine Åhsberg, Emma Rörby, Wan Man Wong and Jan-Ingvar Jönsson, Coexpression of hyperactivated AKT1 with additional genes activated in leukemia drives hematopoietic progenitor cells to cell cycle block and apoptosis, 2015, Experimental Hematology, (43), 7, 554-564.

http://dx.doi.org/10.1016/j.exphem.2015.04.007

Copyright: Elsevier

http://www.elsevier.com/

Postprint available at: Linköping University Electronic Press $\underline{\text { http://urn.kb.se/resolve?urn=urn:nbn:se:liu:diva-120213 }}$ 


\section{Coexpression of hyperactivated AKT1 with additional genes activated in leukemia}

drives hematopoietic progenitor cells to cell cycle block and apoptosis

Yanjuan Tang ${ }^{1}$, Camilla Halvarsson ${ }^{1,2}$, Amanda Nordigården ${ }^{1}$, J. Komal Kumar ${ }^{1}$, Josefine Åhsberg ${ }^{1}$, Emma Rörby ${ }^{1}$, Wan Man Wong ${ }^{3}$, and Jan-Ingvar Jönsson ${ }^{1,2}$

${ }^{1}$ Department of Clinical and Experimental Medicine, Linköping University, SE-58183 Linköping, Sweden

${ }^{2}$ Linköping Integrative Regenerative Medicine Centre, Linköping University, Sweden

${ }^{3}$ Lund Strategic Center for Stem Cell Biology and Cell Therapy, Lund University, SE-221 84 Lund, Sweden

Running title: Pro-apoptotic function of hyperactivated AKT1

Corresponding author: Jan-Ingvar Jönsson, Ph.D., Department of Clinical and Experimental Medicine, Linköping University, SE-58183 Linköping, Sweden

Phone: +46-13-282300; Fax: +46-10-101375; E-mail: jan-ingvar.jonsson@liu.se

Table of Contents category: Malignant Hematopoiesis

Word count: Abstract, 245; Main text: 3,596 


\section{ABSTRACT}

The phosphatidylinositol 3-kinase (PI3K)/AKT pathway is an integral component of signaling involved in the development of many cancers including myeloid leukemias, such as chronic myeloid leukemia (CML) and acute myeloid leukemia (AML). Increased AKT1 activity is frequently seen in AML patients providing leukemic cells with growth and survival promoting signals. An important aspect of AKT1 function is its involvement in cellular metabolism and energy production. Under some circumstances strong activation of AKT1 increases oxidative stress that can cause apoptosis when cells progressively build up excess of free radicals. This has been described in hematopoietic cells overexpressing activated AKT1, however, whether this is true in cells coexpressing other genetic events involved in leukemia is not known. This prompted us to investigate the effect of constitutively active AKT1 (myristoylated AKT1; myrAKT) in hematopoietic progenitor cells expressing constitutively active STAT5, FLT3-ITD, or anti-apoptotic Bcl-2. Surprisingly, myrAKT1 was incompatible with both STAT5- and FLT3-ITD-driven proliferation, which triggered cell cycle block and apoptosis. Moreover, transplantable cells of Bcl-2 transgenic mice were impaired in their engraftment ability to recipient mice when expressing hyperactivated AKT1. This was linked to AKT1-mediated pro-apoptotic functions and not to impairment in homing to the bone marrow. While cells expressing hyperactivated AKT1 displayed higher levels of reactive oxygen species both in vitro and in vivo, the addition of the antioxidant N-acetyl-L-cysteine (NAC) significantly reduced apoptosis. Taken together, the results indicate that constitutive AKT1 activity is incompatible with growth- and survival-promoting ability of other activated genes in AML.

Keywords: Hematopoiesis, mouse, oncogenes, apoptosis, AKT, FLT3-ITD 


\section{INTRODUCTION}

Aberrant signal transduction enhances survival and proliferation of hematopoietic progenitor cells in acute myeloid leukemia (AML). This occurs through various genetic alterations affecting signaling, for instance Fms-like tyrosine kinase-3 (FLT3). Constitutive activation of signaling proteins is frequently demonstrated in AML, comprising major signaling cascades such as the Mitogen-activated protein kinase (MAPK) pathway, the Phosphatidylinositol 3kinase (PI3K)/AKT pathway, and Signal Transducer and Activator of Transcription 5 (STAT5). As these mutant signaling proteins are frequently present in AML they have developed as attractive therapeutic targets.

PI3K is a major signaling protein involved in cell growth, survival, and metabolism. Many of the effects are mediated by the serine/threonine kinase AKT (protein kinase B, PKB) downstream of PI3K. The AKT family comprises AKT1, AKT2, and AKT3 $(\mathrm{PKB} \alpha, \beta$, and $\gamma)$, which are mostly redundant in function. AKT1 is more ubiquitously expressed, whereas AKT2 and AKT3 are restricted to certain tissues. In hematopoietic cells, both AKT1 and AKT2 share important functions although AKT1 seems to be more important in signaling. AKT activation inhibits apoptosis through a variety of mechanisms, including phosphorylation and inhibition of glycogen synthase kinase-3 (GSK-3), the pro-apoptotic Bcl2 family member Bad, caspase-9, and members of the FoxO subfamily of forkhead transcription factors [1].

Dysregulation of the PI3K/AKT pathway has been implicated in many human cancers. The p110 $\alpha$ catalytic subunit of PI3K encoded by PIK3CA is one of the most frequently mutated genes in human cancers and promotes carcinogenesis by activation of AKT1 [2]. Mutations in the Akt1 gene have been detected in some tumors as an alternative pathway of transformation [3, 4]. Contrary, studies of AML indicate no recurrent imbalanced regions within the PIK3CA or Akt1 genes [5-7]. Despite lack of evidence for activating 
mutations of Akt1 in AML, elevated levels of phosphorylated AKT1 have been observed in primary blast cells of most patients [8-10]. The cause of this AKT1 activation remains elusive, but appears linked to upstream signaling events or crosstalk to other pathways. Mutations in the FLT3 gene are one of the most common genetic alterations found in AML patients. Constitutively activated FLT3 occurs most often as internal tandem duplications (ITDs) within the juxtamembrane domain and/or the first kinase domain, and is observed in approximately $20-25 \%$ of all AML patients $[11,12]$. FLT3-ITD mutations are associated with activation of the PI3K/AKT pathway similar to wildtype (WT) FLT3 [13, 14]. In contrast, STAT5 is phosphorylated by FLT3-ITD and not WT FLT3, which is required for transformation in vivo $[15,16]$.

The importance of phosphorylated STAT5 for hematopoietic malignancies is underlined by observations in lymphoid and myeloid leukemias that have constitutive STAT5 phosphorylation. The introduction of constitutively active STAT5 (caSTAT5) mutants into murine hematopoietic cells suffices to induce multilineage leukemia in mice [17]. In contrast, AKT1 overexpression or constitutive active forms of AKT1 are unable to transform hematopoietic cells in vitro [18], but may in combination with other pathways mediate transformation. When activated AKT1 was expressed in a murine bone marrow (BM) transplantation model, recipient mice developed myeloproliferative disease, T-cell lymphoma, or AML [19]. Analysis of hematopoietic stem cells (HSCs) in these mice revealed increased cycling and apoptosis of the transplanted cells as well as impaired engraftment, implying that HSCs are sensitive to excess AKT signaling.

It was recently reported that permanent AKT1 activation can lead to increased oxidative stress that renders cells susceptible to reactive oxygen species (ROS)-triggered apoptosis $[20,21]$. In these studies, AKT1 was overexpressed without the presence of other genetic alterations as expected in primary leukemic cells. This raises questions to the function 
and involvement of activated AKT1 in leukemogenesis when coexpressed with other genes involved in leukemia. Herein we present evidence that constitutively activated AKT1 (myristoylated AKT1) forces hematopoietic progenitors into a quiescent state of cell cycle and induces apoptosis when coexpressed with caSTAT5, FLT3-ITD mutation, or antiapoptotic Bcl-2. Transplanted cells from Bcl-2 transgenic mice expressing constitutively active AKT1 were impaired in engraftment of recipient mice. While cells expressing active AKT1 displayed higher levels of ROS, the addition of the antioxidant N-acetyl-L-cysteine (NAC) significantly reduced apoptosis. These findings suggest that increased oxidative stress by hyperactivated AKT1 is detrimental to hematopoietic cells. Thus, it is possible that enhancing AKT1 activity could lead to strategies in eradicating leukemic cells, even in the presence of other oncogenes or activated pathways. 


\section{MATERIALS AND METHODS}

Mice

C57BL6/J (WT; CD45.2), B6.SJL (CD45.1) or C57BL6/J-vav-Bcl-2 (Bcl-2) mice [22] were housed in the Animal Facility at Linköping University. BM of FLT3-ITD ${ }^{+/-}$mice (B6.129Flt $3^{\text {tmlDgg} / J) ~[23] ~ w a s ~ p r o v i d e d ~ b y ~ E w a ~ S i t n i c k a ~(L u n d, ~ S w e d e n) . ~ T h e ~ s t u d y ~ w a s ~ a p p r o v e d ~ b y ~}$ the Animal ethics committee at Linköping university and carried out in accordance with Swedish legislation for care and use of laboratory animals.

\section{Cell lines and Western blots}

$\mathrm{Ba} / \mathrm{F} 3$ cells were cultured in RPMI 1640 medium (PAA laboratories, Les Mureaux, France) with $10 \%$ fetal calf serum (FCS; Hyclone, Logan, UT, USA), 25 nM Hepes, $50 \mu \mathrm{M} 2-$ mercaptoethanol (Sigma-Aldrich, St Louis, MO), whereas FDCP-1 cells were cultured in Iscove’s modified Dulbecco's medium (IMDM; PAA) with 10\% FCS and 2 mM L-glutamine. Both cell lines were supplemented with $1 \%$ penicillin-streptomycin and 5\% interleukin-3containing supernatant when routinely maintained. Western blots were done with pAKT1 (Ser ${ }^{473}$ ) and total AKT (Cell Signaling Technology, Danvers, MA)

\section{Isolation and culture of $\mathrm{c}-\mathrm{kit}^{+}$cells}

Mononuclear BM cells were harvested by crushing femurs and tibiae from 8-14 weeks old mice. MACS immunomagnetic cell separation using magnetically labeled anti-CD117 beads (Miltenyi, Cologne, Germany) were used to isolate c-kit positive cells. After selection, cells were cultured in IMDM supplemented with $20 \%$ FCS, $10 \mathrm{U} / \mathrm{mL}$ penicillin/streptomycin, 50ng/mL murine stem cell factor (SCF), human interleukin-6 (IL6) and human thrombopoietin (TPO) (all from Peprotech Inc., Rocky Hill, NJ) before retroviral infections. 


\section{Retroviral infections}

Transduction with caSTAT5 and/or myrAKT (myristoylated version of the human Akt1 gene) were done by spinoculation with retrovirus supernatants of pMX-puro-caSTAT5 [24] and pLZRS-myrAKT1-EGFP [21]. As controls, empty vectors were used. Retroviral supernatants were produced by transient transfection of $293 \mathrm{~T}$ cells using calcium phosphate coprecipitation with $4 \mu \mathrm{g}$ cDNA. Cells were plated in $3.5-\mathrm{cm}$ dishes, and $2.5 \mathrm{~mL}$ IMDM medium was refreshed 18 hours after transfection. After an additional 24 and 48 hours, viral supernatants were collected, pooled, and filtered through a $0.45 \mu \mathrm{m}$ filter. Transduction was performed by spinoculation for 90 minutes at $1,800 \mathrm{~g}$ in 24 -well plates in $0.5 \mathrm{~mL}$ of retroviral supernatant and $0.5 \mathrm{~mL}$ IMDM medium supplemented with 20\% FCS, 50ng/mL of SCF, IL6, and TPO each, and 5 $\mathrm{g} / \mathrm{mL}$ polybrene (American Bioanalytical, Natick, MA). When c-kit ${ }^{+}$ cells of FLT3-ITD mice were used, IL-6 was substituted by human FLT3 ligand (FL). Three days posttransduction, $\mathrm{GFP}^{+}$cells were sorted on FACS Aria (BD Biosciences) and cultured in IMDM with $20 \%$ FCS in the presence or absence of cytokines as indicated.

\section{Phosflow analysis}

Cells were stimulated with IL-3 (50 ng/mL) for 30 minutes, then fixed with $1 \%$ paraformaldehyde (EMS, Hatfield, PA) for 10 minutes and permeabilized using $0.3 \%$ saponin (Sigma-Aldrich) in PBS for 45 minutes on ice followed by incubation with ice-cold methanol for 30 minutes on ice. After washing cells were stained with anti-phospho-AKT antibody (pS473, BD Biosciences) conjugated with Alexa-647 for flow cytometry on FACS Canto (BD Biosciences). Acquired data was analyzed on Cytobank (www.cytobank.org). 


\section{Cell cycle analysis}

Cultured cells were fixed with Cytofix/Cytoperm (BD Biosciences) for 30 minutes at $4^{\circ} \mathrm{C}$, followed by washing twice with Perm/Wash (BD Biosciences) buffer. After washing, cells were stained with PE-conjugated anti-Ki67 antibody (BD Biosciences) and DAPI $(0.5 \mu \mathrm{g} / \mathrm{mL}$; Sigma-Aldrich). Cell-cycle status was determined using DAPI versus Ki-67 on FACSAria SORP (BD Biosciences), and the amount of cells in $\mathrm{G}_{0} / \mathrm{G}_{1}$ and $\mathrm{S}$-phase was calculated using FlowJo software (TreeStar Inc., Ashland, OR).

\section{Apoptosis assays}

For apoptosis measurements, cells were collected at the indicated time points, washed with cold PBS+5\% FCS twice, re-suspended in Annexin-V binding buffer, and incubated with Annexin V-APC and 7-aminoactinomycin D (7AAD, $5 \mu \mathrm{g} / \mathrm{mL}$ : both from BD Biosciences) at room temperature for 15 minutes. Cells were analyzed on FACS Canto (BD Biosciences) within an hour. Acquired data was analyzed by FlowJo software.

\section{Transplantations}

Before transplantation, eight- to twelve-week-old CD45.1 mice were lethally irradiated with 9Gy. For intravenous transplantation recipient mice received 10,000 sorted $\mathrm{GFP}^{+}$cells of CD45.2 mice via tail vein injections in $200 \mu$ l. For intrafemoral transplantations, after subcutaneous injection with Temgensic $(0.1 \mathrm{mg} / \mathrm{kg})$, a hole was drilled of the right femur by a $30 \mathrm{G}$ needle (BD Biosciences), after which 5,000 $\mathrm{GFP}^{+}$sorted CD45.2 $2^{+}$cells were injected directly in the BM cavity by a Hamilton syringe with a $31 \mathrm{G}$ needle (Hamilton Bonaduz AG, Switzerland) in $10 \mu 1$. After transplantation, mice were maintained under sterile conditions in microisolater cages and provided with autoclaved food and water containing $111 \mathrm{mg} / \mathrm{L}$ ciprofloxacin (Ciproxin: Fresenius Kabi, Uppsala, Sweden). Peripheral blood was collected 
by lateral tail vein bleeding and analyzed $1,2,4,8$, or 12 weeks posttransplant, depending on the experiment, by tracking CD45.1, CD45.2, and GFP expression on FACS Canto using antiCD45.1-PE and anti-CD45.2-PE-Cy7 (BioLegend, San Diego, CA). Erythrocytes were lysed with ammonium chloride (Stem Cell Technologies), and the leukocytes resuspended in PBS with 5\% FCS. Data were analyzed using FlowJo software.

\section{Measurements of ROS}

ROS were measured by flow cytometry as indicated by the manufacturers using either $10 \mu \mathrm{M}$ 6-carboxy-2',7'-dichlorodihydrofluorescein diacetate (carboxy- $\mathrm{H}_{2}$ DCFDA) (Molecular Probes, Eugene, ON) or CellROX ${ }^{\circledR}$ Deep Red Flow Cytometry assay kit (Life Technologies, Waltham, MA). ROS activity was inhibited by the addition of $\mathrm{N}$-acetyl-cysteine (NAC; Sigma-Aldrich).

\section{Statistical analysis}

Statistical testing was performed using InStat software (Graphpad Software, San Diego, CA). Experiments performed with sorted $\mathrm{GFP}^{+}$cells were done at least in triplicate. Data were expressed as mean values and SD. Statistical significance of differences observed between groups were determined by Student's t-test. 


\section{RESULTS}

\section{Constitutively active AKT1 is incompatible with STAT5-driven proliferation}

To elucidate whether hyperactivated AKT1 causes apoptosis in hematopoietic cells coexpressing additional oncogenes present in primary AML cells, we overexpressed myrAKT together with constitutively active STAT5 (caSTAT5), mutated FLT3-ITD, or anti-apoptotic Bcl-2 in hematopoietic progenitors. STAT5 is an indispensable downstream target in leukemia and cells transformed by bcr-abl [30, 31] or FLT3-ITD [16, 32, 33] are addicted to STAT5 for maintaining the leukemic state. In this study, we have used myristoylated AKT1 (myrAKT) to mimic constitutively activated AKT1. When fused to the Src myristylation signal, AKT1 is targeted to the cell membrane where it becomes activated and phosphorylated [25], as demonstrated in the hematopoietic progenitor cell line FDCP-1 (Figure 1a).

We transduced cells by retroviral gene transfer with myrAKT1 and/or caSTAT5 into two IL-3-dependent hematopoietic progenitor cell lines: Ba/F3 pro B-cells and myeloid FDCP-1 cells. In agreement with previous reports [26, 27], myrAKT1 was insufficient for cell growth and survival of Ba/F3 cells deprived of cytokines, whereas caSTAT5 sustained proliferation and cells increased in numbers during the five days analyzed (Figure 1b). Contrary, hyperactivated AKT1 disabled STAT5-mediated proliferation (Figure 1b). This was also true in FDCP-1 (Figure 1c). To generate high numbers of transduced cells for analysis, FDCP-1 cells were first selected in puromycin in the presence of IL-3 for four days and then tested for effects of caSTAT5 and myrAKT after IL-3 starvation. The same effect of myrAKT as with BaF3 was seen, thus hyperactivated AKT1 suppressed STAT5-mediated proliferation and cell viability. These results are surprising since previous studies have implied that both AKT1 and STAT5 can be simultaneously activated in leukemic cells, for instance via FLT3ITD [28, 29]. 
Constitutively active AKT1 induces cell cycle block and apoptosis of FLT3-ITD expressing bone marrow progenitor cells

Bearing in mind that AKT1 and STAT5 are downstream targets of FLT3-ITD, but myrAKT1 was incompatible with caSTAT5-driven proliferation, we decided to study the consequence of overexpressing myrAKT1 in hematopoietic progenitors from BM of FLT3-ITD transgenic mice. We first determined whether myrAKT1 was phosphorylated in transduced cells. Western blot (Figure 2a) and Phosflow analyses (Figure 2b) of myrAKT1-transduced cells showed that AKT1 was phosphorylated in the absence of external stimulus as strong as endogenous AKT1 protein in IL-3-stimulated control cells (WT/Puro). Expression of FLT3ITD in progenitor cells induced constitutive AKT1 phosphorylation, and coexpression of myrAKT led to even stronger phosphorylation (Figure 2a-b).

Next we transduced c-kit ${ }^{+}$BM progenitor cells from FLT3-ITD transgenic mice with myrAKT1/GFP in the presence of cytokines (SCF+FL or SCF+TPO+FL). Two days after infection, $\mathrm{GFP}^{+}$cells were FACS-sorted and re-cultured with or without cytokines and analyzed for cell cycle status and Annexin V/7-AAD staining for apoptosis. In the absence of cytokines the proportion of cells in $\mathrm{G}_{0}$ from WT mice after 48 hours increased to $46.6 \%$ when expressing myrAKT1 compared to control GFP cells displaying only $18.8 \%$ cells in $\mathrm{G}_{0}$ (Figure 2c). This increased further to $66.4 \%$ when myrAKT1 was expressed in progenitor cells of FLT3-ITD mice. Surprisingly, this was also seen when cells were cultured with cytokines. In this case, the numbers of ${\mathrm{c}-\mathrm{kit}^{+}}^{+}$progenitors in $\mathrm{G}_{0}$ after stimulation with $\mathrm{SCF}+\mathrm{FL}$ increased to $53.1 \%$ in FLT3-ITD cells expressing myrAKT1 compared to $22.8 \%$ in control GFP-infected cells. Similar myrAKT1-infected cells of FLT3-ITD mice cultured in $\mathrm{SCF}+\mathrm{TPO}+\mathrm{FL}$ accumulated in $\mathrm{G}_{0}$ to $59.7 \%$ compared to $31.9 \%$ for control cells. With some delay (72 hours) the numbers of apoptotic cells, detected as Annexin V/7-AAD staining of cells, increased to some extent (20-25\%) in cultures of cells expressing myrAKT1 compared 
to GFP-infected cells (Figure 2d), indicating that AKT1-driven cell cycle exit and apoptosis is not abrogated by the strong pro-survival and proliferative activity of FLT3-ITD.

\section{Hyperactivated AKT1 induces apoptosis in progenitor cells expressing Bcl-2}

FLT3-ITD $^{+}$cell lines and primary cells from AML patients display elevated levels of the antiapoptotic Bcl-2 family member myeloid cell leukemia-1 (MCL-1). Furthermore, MCL-1 is dependent of FLT3-ITD signaling since the expression is reversible upon pharmacological inhibition of FLT3 activity by tyrosine kinase inhibitors [34]. Recently, it was observed that FLT3-ITD-dependent up-regulation of MCL-1 was linked to STAT5 [33]. Considering our results that myrAKT1 interferes with STAT5- and FLT3-ITD-driven proliferation, we decided to coexpress myrAKT1 with anti-apoptotic Bcl-2. Progenitor cells from BM of Bcl-2 mice were transduced with myrAKT1/GFP, and after sorting $\mathrm{GFP}^{+}$cells, cell cycle and apoptosis during 72 hours were analyzed. After 24 hours, Bcl-2 cells coexpressing myrAKT1 accumulated to a higher extent in $\mathrm{G}_{0}$ of cell cycle when grown without cytokines compared to cells infected with control virus (31.4\% versus $17.6 \%$; Figure $3 a)$. This was followed by increased numbers of apoptotic cells as measured by Annexin-V binding and 7-AAD staining after 72 hours with $22.0 \%$ for WT-GFP, $53.5 \%$ for WT-myrAKT1, 30.9\% for Bcl-2/GFP, and $54.9 \%$ for Bcl-2/myrAKT1 ( $\mathrm{n}=3$; Figure $3 \mathrm{~b}$ ). One representative experiment for detection of apoptotic cells as measured as Annexin-V binding and 7-AAD staining is shown in Figure 3c. The results demonstrate that Bcl-2 fails to rescue cell death by hyperactivated AKT1.

\section{Hyperactivated AKT1 impairs engraftment of Bcl-2 expressing progenitor cells}

Next we decided to test whether myrAKT1 overexpression have pro-apoptotic effects in vivo. Recipient mice were transplanted with BM progenitor cells enriched for c-kit expression from WT or Bcl-2 transgenic mice transduced with myrAKT1/GFP or GFP control virus. Forty- 
eight hours post-infection, $1 \times 10^{4} \mathrm{GFP}^{+}$cells were injected to lethally irradiated mice in a noncompetitive setting. Peripheral blood was then analyzed for GFP expression after 4, 8, and 12 weeks. Already after 4 weeks reconstitution was negatively affected in mice receiving myrAKT1-expressing BM cells. The level of donor cell reconstitution in peripheral blood was $54.9 \%$ for WT/GFP, $17.1 \%$ for WT/myrAKT, $67.2 \%$ for Bcl-2/GFP, and 25.2\% for Bcl2/myrAKT (Figure 4a). At longer time points reconstitution remained high in mice transplanted with GFP control virus but was severely impaired in mice transplanted with myrAKT1-expressing cells (Figure 4a). Several of these mice succumbed to hematopoietic failure during the first 8 weeks (Figure 4b).

Impaired engraftment of bone marrow cells expressing hyperactivated AKT1 does not correlate to disturbed homing

Previous studies have demonstrated that AKT1 can regulate trafficking of hematopoietic progenitors by influencing expression of adhesion molecules and homing receptors [21]. Since we could not distinguish whether the effects of myrAKT1 was due to apoptotic induction or the ability of AKT1 to disable homing and/or adhesion of progenitor cells to the $\mathrm{BM}$, we decided to compare intravenous injections to direct delivery to the BM by intrafemoral injections. As seen in Figure 4c, engraftment was impaired when myrAKT1expressing cells were injected directly to the marrow, demonstrating that hyperactivated AKT1 negatively affected hematopoietic reconstitution but not by homing defects.

Hyperactivated AKT1 increases the levels of intracellular ROS but antioxidant NAC protects cells from activated AKT1-induced apoptosis

Previous studies have shown that activation of AKT1 can lead to accumulated levels of intracellular ROS, which could be detrimental to hematopoietic cells [26]. To investigate 
whether this was the cause of the pro-apoptotic effects mediated by activated AKT1 in cells expressing FLT3-ITD, STAT5, or Bcl-2, we analyzed ROS levels in cells overexpressing myrAKT1. To measure the ROS levels, cells infected with a puromycin-containing myrAKT1 virus were stained with DCFDA and analyzed by flow cytometry. In cells infected with myrAKT1, the levels of ROS increased significantly 48 hours postselection with puromycin (Figure 5a). Treatment of infected $\mathrm{GFP}^{+}$FACS-sorted cells with NAC, a well-known antioxidant, prevented apoptosis partially (Figure 5b-c), suggesting that the detrimental effects by hyperactivated AKT1 is linked to accumulation of increased levels of ROS. This was further confirmed in myrAKT1/GFP-transplanted progenitor cells from wildtype mice after injection to recipient mice. Seven days posttransplant $\mathrm{GFP}^{+}$donor cells in peripheral blood were analyzed for ROS level by staining with CellRox Deep Red and flow cytometry. Cells with myrAKT1 in recipient mice expressed two-fold higher ROS level than cells transduced with control virus (Figure 5d). 


\section{DISCUSSION}

Despite evidence of elevated levels of phosphorylated AKT1 in primary leukemic blast cells, reoccurring mutations in the Akt1 gene are absent in patients with AML, raising the question whether permanent AKT1 activation is incompatible with leukemogenesis. As a model to investigate the consequence of hyperactivated AKT1 in hematopoietic cells expressing additional oncogenic events, we used FLT3-ITD signaling, present in approximately one-forth of all AML patients, as a model. We used a myristoylated form of AKT1 that is localized at the cell membrane and becomes constitutively activated, where the protein is expected to execute normal signaling functions. Our data demonstrate that myrAKT1 disables STAT5mediated proliferation of Ba/F3 and FDCP-1 cells. In addition, myrAKT1 had detrimental effects on cell proliferation and induced apoptosis when overexpressed in BM-derived progenitors from FLT3-ITD transgenic mice. Although a cell cycle block was more evident in cells cultured in the absence of cytokines, FLT3-ITD cells coexpressing myrAKT1 cultured in cytokines displayed significantly more cells in $\mathrm{G}_{0}$ after 24 hours in culture and were approximately $20 \%$ more apoptotic after 72 hours. This indicates that AKT1-driven cell cycle block and apoptosis is not inhibited by FLT3-ITD activity. Although AKT1 is a downstream target of FLT3-ITD, we interpret the results as an overstimulation of cells expressing both FLT3-ITD and hyperactivated AKT1. Other previous reports have indicated that AKT1 may facilitate rather than inhibit cell death under certain conditions [20, 35, 36]. However, our data are novel with regard to the pro-apoptotic function of AKT1 in the presence of other growthpromoting and anti-apoptotic oncogenic events.

There are other examples of oncogenes promoting proliferation that trigger apoptosis when delivering a strong mitogenic signal, such as c-MYC [37]. However, in contrast to the concept that constitutive oncogenic-driven apoptosis is due to cell-cycle entry sensitizing cells to apoptosis, myrAKT1 together with the three oncogenes we have tested 
(caSTAT5, FLT3-ITD, and Bcl-2) led to cell cycle block prior to cell death. The reason for this is presently not known.

Previous reports have indicated that pro-apoptotic function of hyperactivated AKT1 could be linked to suppression of antioxidant enzymes and an increase in ROS levels $[20,38]$. In our study, hematopoietic progenitor cells expressing myrAKT1 contained higher levels of ROS both in vitro and in vivo, which was counteracted in vitro by addition of NAC known as a scavenger of excess levels of ROS. A recent study in mice double deficient of AKT1 and AKT2 indicated that HSCs lacking AKT function persisted in $\mathrm{G}_{0}$ and that the intracellular content of ROS was decreased [39]. This implicates that AKT1 regulates ROS for proper HSC maintenance and differentiation. It is also possible that AKT1 hyperactivity has different effects in progenitor cells, driving them towards cell cycle block due to harmful effects by increased ROS levels. For instance, Ba/F3 cells expressing hyperactivated AKT1 are short-lived upon growth factor-deprivation and are induced to apoptosis [26].

The inability of Bcl-2 to prevent myrAKT1-mediated pro-apoptotic effects is surprising considering previous findings that $\mathrm{Bcl}-2$ is involved as an antioxidant agent to overcome ROS effects [40]. However, in previous studies where AKT1 has been shown to have harmful effects $[20,26]$, AKT1 was expressed as a fusion protein to the estrogen receptor (ER). AKT1 activity was then controlled by tamoxifen treatment where the ERfusion protein translocated to the nucleus [26]. In contrast, myrAKT1 mimics the novel Akt1 pleckstrin homology domain (PHD) mutation (AKT1-E17K) found in solid tumors and is localized at the cell membrane [41]. This could mean that AKT1 executes different functions when nuclear localized versus targeted to the cell membrane.

In summary, several studies suggest that AKT1 is harmful to hematopoietic cells when constitutively expressed. Here we show that hyperactivity of AKT1 is harmful by an improper balance of ROS. Although presently unknown this could be one reason why 
activating mutations of $A k t 1$ leading to permanent activation are absent in AML in contrast to several other cancers. Phosphorylation and activation of AKT1 is under tight regulation where the AKT1 protein is transiently oscillating between active and inactive states [42]. Since activating mutations of FLT3-ITD and other signaling proteins in leukemia activate the PI3K/AKT pathway, the results that hyperactivated AKT1 can lead to apoptosis could lead to future drug development to eradicate leukemic cells in AML patients by enhancing AKT1 activity. 


\section{CONFLICT OF INTEREST}

The authors declare no conflict of interest.

\section{ACKNOWLEDGEMENTS}

We thank Pia Druid for expert technical help. We also thank Drs. Jerry Adams (Melbourne, Australia) and Ewa Sitnicka (Lund, Sweden) for mouse strains, and Drs. Paul Coffer (Utrecht, Netherlands) and Mikael Sigvardsson (Linköping, Sweden) for constructs. This work was supported by grants from the Swedish Research Council, Swedish Cancer Foundation, Swedish Children's Cancer Foundation, County Council of Östergötland, the Faculty of Medicine at Linköping University, and the Ollie and Elof Ericssons Foundation. 


\section{REFERENCES}

1. Martelli, A.M., et al., The emerging role of the phosphatidylinositol 3kinase/Akt/mammalian target of rapamycin signaling network in normal myelopoiesis and leukemogenesis. Biochim Biophys Acta, 2010. 1803(9): p. 991-1002.

2. Samuels, Y., et al., High frequency of mutations of the PIK3CA gene in human cancers. Science, 2004. 304(5670): p. 554.

3. Davies, M.A., Regulation, role, and targeting of Akt in cancer. J Clin Oncol, 2011. 29(35): p. 4715-7.

4. Bleeker, F.E., et al., AKT1(E17K) in human solid tumours. Oncogene, 2008. 27(42): p. 5648-50.

5. Hummerdal, P., et al., Absence of hot spot mutations of the PIK3CA gene in acute myeloid leukaemia. Eur J Haematol, 2006. 77(1): p. 86-7.

6. Muller, C.I., et al., Rare mutations of the PIK3CA gene in malignancies of the hematopoietic system as well as endometrium, ovary, prostate and osteosarcomas, and discovery of a PIK3CA pseudogene. Leuk Res, 2007. 31(1): p. 27-32.

7. Tibes, R., et al., PI3K/AKT pathway activation in acute myeloid leukaemias is not associated with AKT1 pleckstrin homology domain mutation. Br J Haematol, 2008. 140(3): p. 344-7.

8. Gallay, N., et al., The level of AKT phosphorylation on threonine 308 but not on serine 473 is associated with high-risk cytogenetics and predicts poor overall survival in acute myeloid leukaemia. Leukemia, 2009. 23(6): p. 1029-38.

9. Tazzari, P.L., et al., Detection of serine 473 phosphorylated Akt in acute myeloid leukaemia blasts by flow cytometry. Br J Haematol, 2004. 126(5): p. 675-81.

10. Min, Y.H., et al., Cytoplasmic mislocalization of p27Kipl protein is associated with constitutive phosphorylation of Akt or protein kinase $B$ and poor prognosis in acute myelogenous leukemia. Cancer Res, 2004. 64(15): p. 5225-31.

11. Small, D., FLT3 mutations: biology and treatment. Hematology Am Soc Hematol Educ Program, 2006: p. 178-84.

12. Kayser, S., et al., Insertion of FLT3 internal tandem duplication in the tyrosine kinase domain-1 is associated with resistance to chemotherapy and inferior outcome. Blood, 2009. 114(12): p. 2386-92.

13. Brandts, C.H., et al., Constitutive activation of Akt by Flt3 internal tandem duplications is necessary for increased survival, proliferation, and myeloid transformation. Cancer Res, 2005. 65(21): p. 9643-50.

14. Jönsson, M., M. Engstrom, and J.I. Jönsson, FLT3 ligand regulates apoptosis through AKT-dependent inactivation of transcription factor FoxO3. Biochem Biophys Res Commun, 2004. 318(4): p. 899-903.

15. Choudhary, C., et al., Activation mechanisms of STAT5 by oncogenic Flt3-ITD. Blood, 2007. 110(1): p. 370-4.

16. Rocnik, J.L., et al., Roles of tyrosine 589 and 591 in STAT5 activation and transformation mediated by FLT3-ITD. Blood, 2006. 108(4): p. 1339-45.

17. Moriggl, R., et al., Stat5 tetramer formation is associated with leukemogenesis. Cancer Cell, 2005. 7(1): p. 87-99.

18. Shelton, J.G., et al., Effects of the RAF/MEK/ERK and PI3K/AKT signal transduction pathways on the abrogation of cytokine-dependence and prevention of apoptosis in hematopoietic cells. Oncogene, 2003. 22(16): p. 2478-92.

19. Kharas, M.G., et al., Constitutively active AKT depletes hematopoietic stem cells and induces leukemia in mice. Blood, 2010. 115(7): p. 1406-15. 
20. Nogueira, V., et al., Akt determines replicative senescence and oxidative or oncogenic premature senescence and sensitizes cells to oxidative apoptosis. Cancer Cell, 2008. 14(6): p. 458-70.

21. Buitenhuis, M., et al., Protein kinase B (PKB/c-akt) regulates homing of hematopoietic progenitors through modulation of their adhesive and migratory properties. Blood, 2010. 116(13): p. 2373-84.

22. Ogilvy, S., et al., Constitutive Bcl-2 expression throughout the hematopoietic compartment affects multiple lineages and enhances progenitor cell survival. Proc Natl Acad Sci U S A, 1999. 96(26): p. 14943-8.

23. Lee, B.H., et al., FLT3 mutations confer enhanced proliferation and survival properties to multipotent progenitors in a murine model of chronic myelomonocytic leukemia. Cancer Cell, 2007. 12(4): p. 367-80.

24. Royer, Y., et al., High-throughput gateway bicistronic retroviral vectors for stable expression in mammalian cells: exploring the biologic effects of STAT5 overexpression. DNA Cell Biol, 2004. 23(6): p. 355-65.

25. Kohn, A.D., F. Takeuchi, and R.A. Roth, Akt, a pleckstrin homology domain containing kinase, is activated primarily by phosphorylation. J Biol Chem, 1996. 271(36): p. 21920-6.

26. van Gorp, A.G., et al., Chronic protein kinase B (PKB/c-akt) activation leads to apoptosis induced by oxidative stress-mediated Foxo3a transcriptional up-regulation. Cancer Res, 2006. 66(22): p. 10760-9.

27. Shelton, J.G., et al., Ability of the activated PI3K/Akt oncoproteins to synergize with MEK1 and induce cell cycle progression and abrogate the cytokine-dependence of hematopoietic cells. Cell Cycle, 2004. 3(4): p. 503-12.

28. Fernandes, M.S., et al., Novel oncogenic mutations of CBL in human acute myeloid leukemia that activate growth and survival pathways depend on increased metabolism. J Biol Chem, 2010. 285(42): p. 32596-605.

29. Masson, K. and L. Ronnstrand, Oncogenic signaling from the hematopoietic growth factor receptors $c$-Kit and Flt3. Cell Signal, 2009. 21(12): p. 1717-26.

30. Hoelbl, A., et al., Stat5 is indispensable for the maintenance of bcr/abl-positive leukaemia. EMBO Mol Med, 2010. 2(3): p. 98-110.

31. Warsch, W., et al., High STAT5 levels mediate imatinib resistance and indicate disease progression in chronic myeloid leukemia. Blood, 2011. 117(12): p. 3409-20.

32. Kiyoi, H., et al., A novel FLT3 inhibitor FI-700 selectively suppresses the growth of leukemia cells with FLT3 mutations. Clin Cancer Res, 2007. 13(15 Pt 1): p. 4575-82.

33. Yoshimoto, G., et al., FLT3-ITD up-regulates MCL-1 to promote survival of stem cells in acute myeloid leukemia via FLT3-ITD-specific STAT5 activation. Blood, 2009. 114(24): p. 5034-43.

34. Kasper, S., et al., Targeting MCL-1 sensitizes FLT3-ITD-positive leukemias to cytotoxic therapies. Blood Cancer J, 2012. 2(3): p. e60.

35. Benbrook, D.M. and C.P. Masamha, The pro-survival function of Akt kinase can be overridden or altered to contribute to induction of apoptosis. Curr Cancer Drug Targets, 2011. 11(5): p. 586-99.

36. Los, M., et al., Switching Akt: from survival signaling to deadly response. Bioessays, 2009. 31(5): p. 492-5.

37. Pelengaris, S., M. Khan, and G. Evan, $c-M Y C$ : more than just a matter of life and death. Nat Rev Cancer, 2002. 2(10): p. 764-76.

38. Esposito, F., et al., Protein kinase B activation by reactive oxygen species is independent of tyrosine kinase receptor phosphorylation and requires SRC activity. $\mathrm{J}$ Biol Chem, 2003. 278(23): p. 20828-34. 
39. Juntilla, M.M., et al., AKT1 and AKT2 maintain hematopoietic stem cell function by regulating reactive oxygen species. Blood, 2010. 115(20): p. 4030-8.

40. Rassool, F.V., et al., Reactive oxygen species, DNA damage, and error-prone repair: a model for genomic instability with progression in myeloid leukemia? Cancer Res, 2007. 67(18): p. 8762-71.

41. Carpten, J.D., et al., A transforming mutation in the pleckstrin homology domain of AKT1 in cancer. Nature, 2007. 448(7152): p. 439-44.

42. Liu, P., et al., Cell-cycle-regulated activation of Akt kinase by phosphorylation at its carboxyl terminus. Nature, 2014. 508(7497): p. 541-5. 


\section{FIGURE LEGENDS}

Figure 1. Expression of activated myrAKT1 is incompatible with caSTAT5-driven proliferation in mouse bone marrow-derived hematopoietic progenitor cells

(a) Western blots of cell lysates from FDCP-1 cells infected with retrovirus containing either WT-AKT1 or myrAKT1 and stimulated for 15 minutes with SCF (100 ng/mL). (b) Number of viable cytokine-deprived $\mathrm{Ba} / \mathrm{F} 3$ cells infected with retrovirus containing puro-caSTAT5 and/or myrAKT1/GFP, determined by trypan blue exclusion. Data represent the average number of cells/mL \pm SD ( $\mathrm{n}=3)$. (c) Number of viable cytokine-deprived FDCP-1 cells infected with retrovirus containing puro-caSTAT5 and/or myrAKT1/GFP. Cells were allowed to grow in the presence of IL-3 $(10 \mathrm{ng} / \mathrm{mL})$ for 4 days after which cells were starved and the number of viable cells were determined by trypan blue exclusion on the days indicated. Data represent the average number of cells $/ \mathrm{mL} \pm \mathrm{SD}(\mathrm{n}=3)$.

Figure 2. myrAKT1 induces cell cycle exit and apoptosis in hematopoietic bone marrow progenitor cells of FLT3-ITD transgenic mice.

(a) Western blot analysis $(\mathrm{n}=2)$ for pAKT1 (pS473) in wildtype or FLT3-ITD ${ }^{+}$progenitor cells transduced with myrAKT1 or control retrovirus either unstimulated or stimulated for 30 minutes with IL-3 (50 ng/mL) to detect endogenous AKT1 phosphorylation. (b) Detection of AKT1 (pS473)-phosphorylation by Phosflow analysis in the same cells as in (a). One representative experiment out of three is shown. (c, d) BM c-kit ${ }^{+}$cells from C57BL6/J WT control or FLT3-ITD ${ }^{+/-}$transgenic mice were cultured overnight before retroviral infections. Forty-eight hours later, $\mathrm{GFP}^{+}$cells were cultured in duplicates without cytokines or with $\mathrm{SCF}+\mathrm{FL}$ or SCF+TPO+FL. (c) After an additional 48 hours, the cell cycle status was analyzed by flow cytometry. Data represent the average number of cells $/ \mathrm{mL} \pm \mathrm{SD}(\mathrm{n}=3)$. (d) Flow cytometric analysis of apoptotic cells (Annexin V binding and 7-AAD staining) after 72 
hours. Data presented as mean $\pm \operatorname{SD}(n=3)$. Statistical analysis was performed using a student's t-test. ${ }^{*} p \leq 0.05, * * p \leq 0.01, * * * p \leq 0.001$.

Figure 3. Activated AKT1 induces cell cycle exit and increases apoptosis of bone marrow

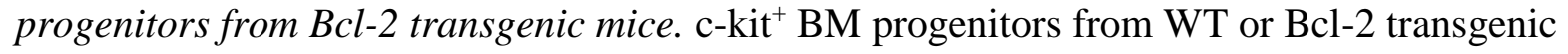
mice were infected with retrovirus containing control GFP or myrAKT1/GFP. Forty-eight hours later, $\mathrm{GFP}^{+}$cells were cultured without cytokines (black bars) or with SCF+TPO+IL-6 (white bars). (a) After 24 hours, cell cycle status was analyzed by flow cytometry. (b) Flow cytometric analysis of apoptotic cells (Annexin V binding and 7-AAD staining) after 72 hours. Data are presented as mean \pm SD $(n=3)$. (c) Representative flow cytometry data of cells stained for Annexin V and 7-AAD. Statistical analysis was performed using a student's t-test. $* * p \leq 0.01, * * * p \leq 0.001$, n.s. $=$ not significant.

Figure 4. Short-term and long-term hematopoietic stem cells coexpressing activated AKT are impaired of engraftment potential even when expressing Bcl-2. (a) Level of engraftment in B6.SJL (CD45.1) recipient mice $(\mathrm{n}=5)$ transplanted with $1 \times 10^{4} \mathrm{c}-\mathrm{kit}^{+} \mathrm{BM}$ progenitor cells of C57BL6/J (CD45.2) from WT or Bcl-2 transgenic mice. Cells were infected for 2 days with control GFP or myrAKT1/GFP retrovirus, and then FACS-sorted for GFP expression before lateral tail injections. The level of reconstitution of donor cells in recipient mice was analyzed for CD45.1, CD45.2, and GFP expression after 4, 8, and 12 weeks by flow cytometry on peripheral blood. Each dot represents one mouse, and the data are presented as mean (horizontal line) \pm SD. (b) Survival curves during 85 days post-transplant of mice $(n=10)$

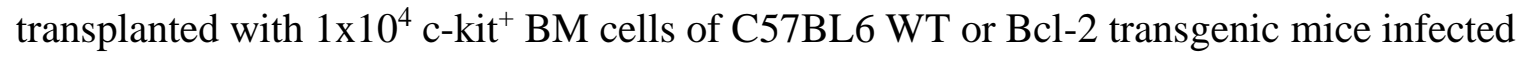
with control GFP or myrAKT1/GFP retrovirus. (c) Engraftment levels as measured two weeks post-transplant for GFP expression in peripheral blood of individual mice $(n=5)$ 
transplanted with c-kit ${ }^{+} \mathrm{BM}$ cells of C57BL6 or Bcl-2 transgenic mice infected with control GFP or myrAKT1/GFP retrovirus injected either by intravenous tail injections or intrafemoral injections. Each dot represents one mouse, and the data are presented as mean (horizontal line). Statistical analysis was performed using a student's t-test. ** $p \leq 0.01, * * * p \leq 0.001$, n.s. $=$ not significant.

Figure 5. Activated AKT1 increases the levels of intracellular ROS but NAC protects cells from activated AKT1-induced apoptosis. (a) c-kit ${ }^{+}$BM progenitors cells of C57BL6 WT or Bcl-2 transgenic mice were infected with a puromycin-retrovirus containing myrAKT1 or empty virus. After 2 days of puromycin selection $(2.0 \mu \mathrm{g} / \mathrm{mL})$, cells were analyzed for ROS by flow cytometry. Data are presented as mean $\pm \mathrm{SD}(\mathrm{n}=3$; in duplicates) of DCFDA mean fluorescence intensity (MFI). (b) c-kit ${ }^{+}$BM progenitors cells of C57BL6 WT or Bcl-2 transgenic mice were infected for 2 days with myrAKT1/GFP retrovirus. GFP ${ }^{+}$cells were recultured with or without 1.0 and $2.0 \mathrm{nM}$ NAC for 72 hours and analyzed for apoptosis (Annexin V binding and 7-AAD staining) by flow cytometry. Data are presented as mean \pm SD ( $n=3)$. (c) Representative flow cytometry data of Annexin V/7AAD-binding of myrAKT1/GFP-transduced cells treated with NAC as in (b). (d) c-kit ${ }^{+}$BM progenitors cells of C57BL6 WT mice were infected with control GFP or myrAKT1/GFP retrovirus and then FACS-sorted for GFP expression before lateral tail injections. ROS level as mean fluorescence intensity was determined in $\mathrm{GFP}^{+}$cells from peripheral blood after 1 week by CellRox Deep Red staining and flow cytometry (WT/GFP: 741112; WT/myrAKT: $1,541 \pm 138)$. Each dot represents one mouse $(n=6)$, and the data are presented as mean (horizontal line) \pm SD. Statistical analysis was performed using a student's t-test. $* p \leq 0.05$ $* * p \leq 0.01, * * * p \leq 0.001$, n.s. $=$ not significant. 
a

$$
\begin{aligned}
& \frac{-}{-+} \frac{\text { mAKT AKT }}{-+} \frac{\text { AKT }}{-+} \text { SCF } \\
& -\cdots \quad \text { pAKT } \\
& \hline==- \text { total AKT }
\end{aligned}
$$

b

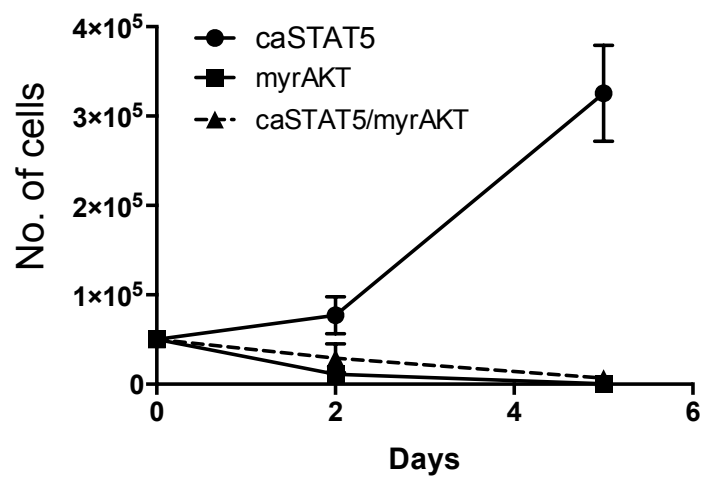

C

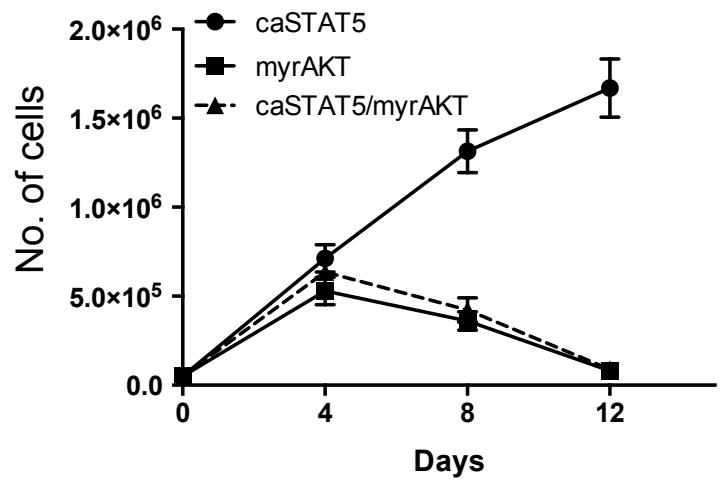

Figure 1 
a

b

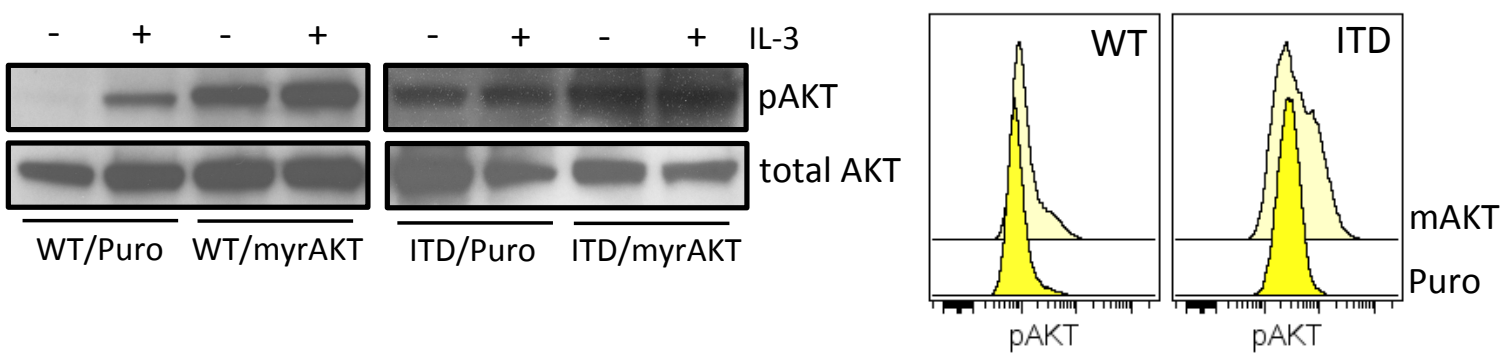

C

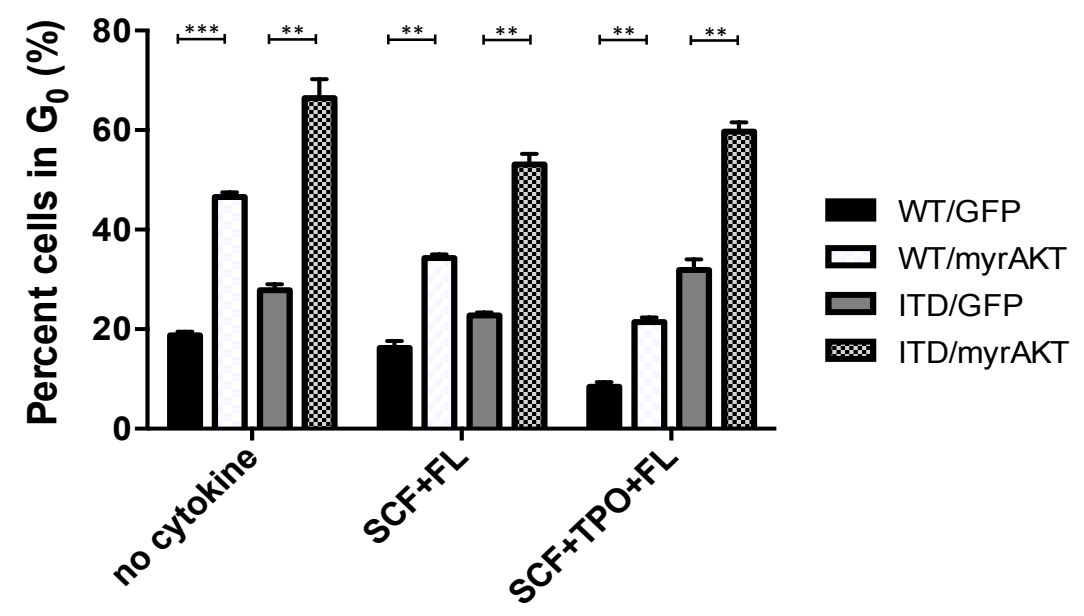

d

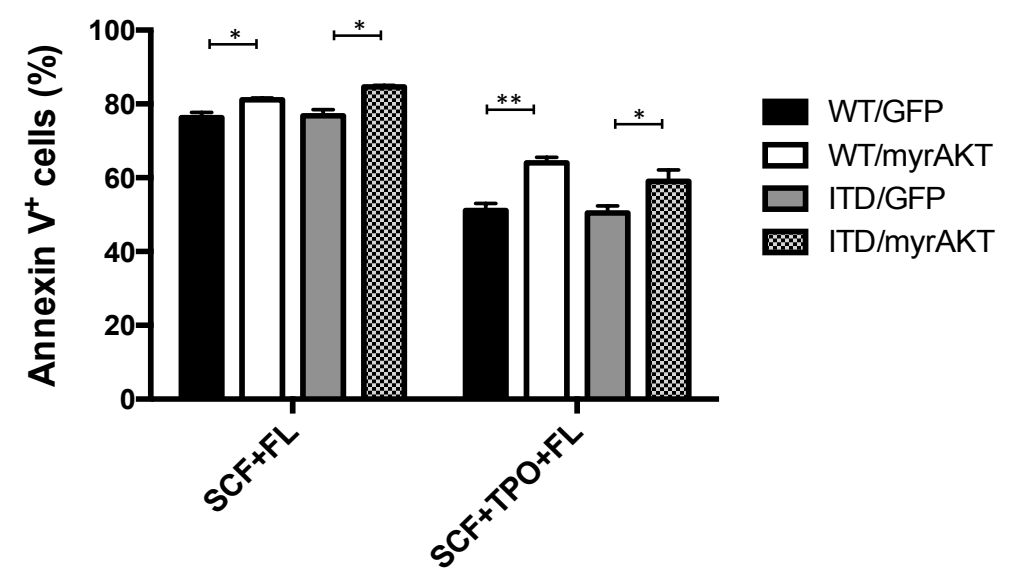

Figure 2 


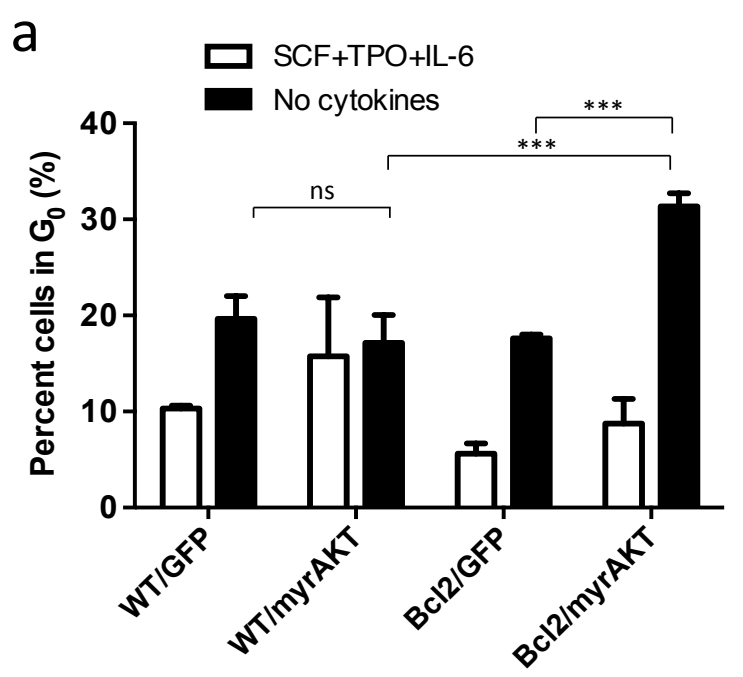

24 hours

b

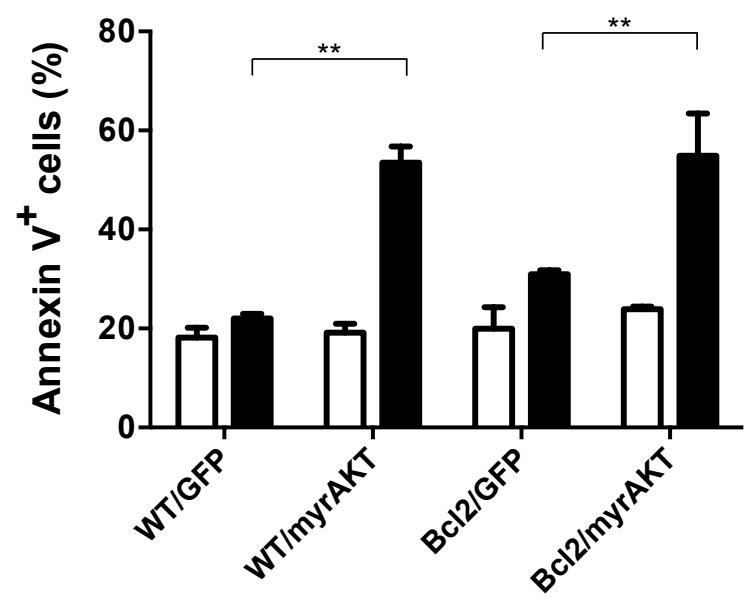

72 hours

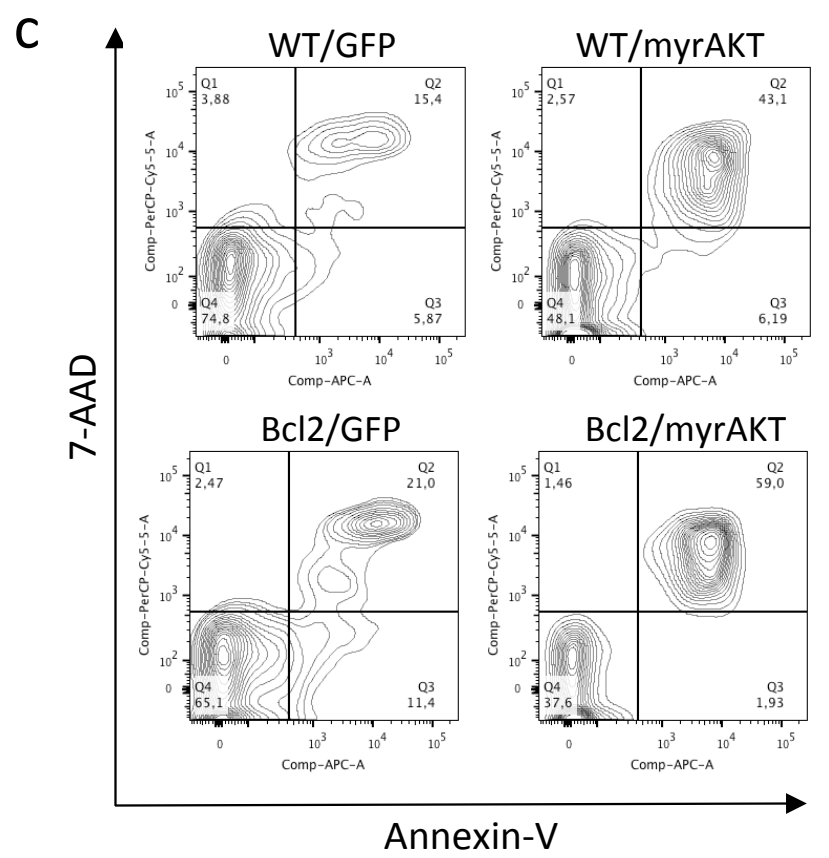

Figure 3 
a

4 weeks

8 weeks

12 weeks
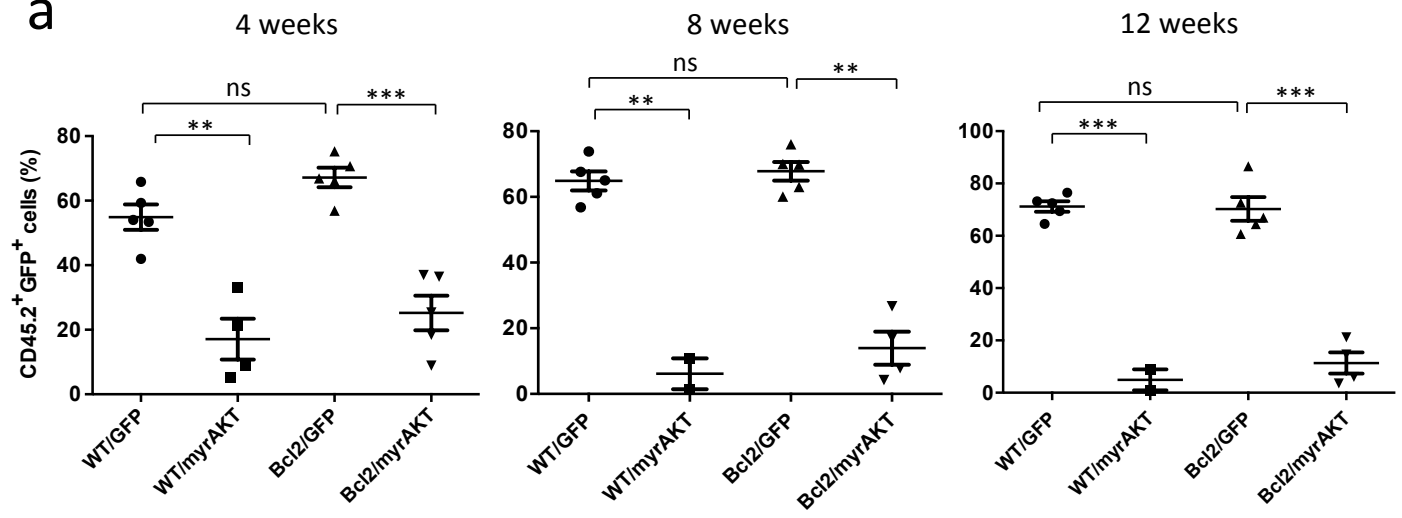

b
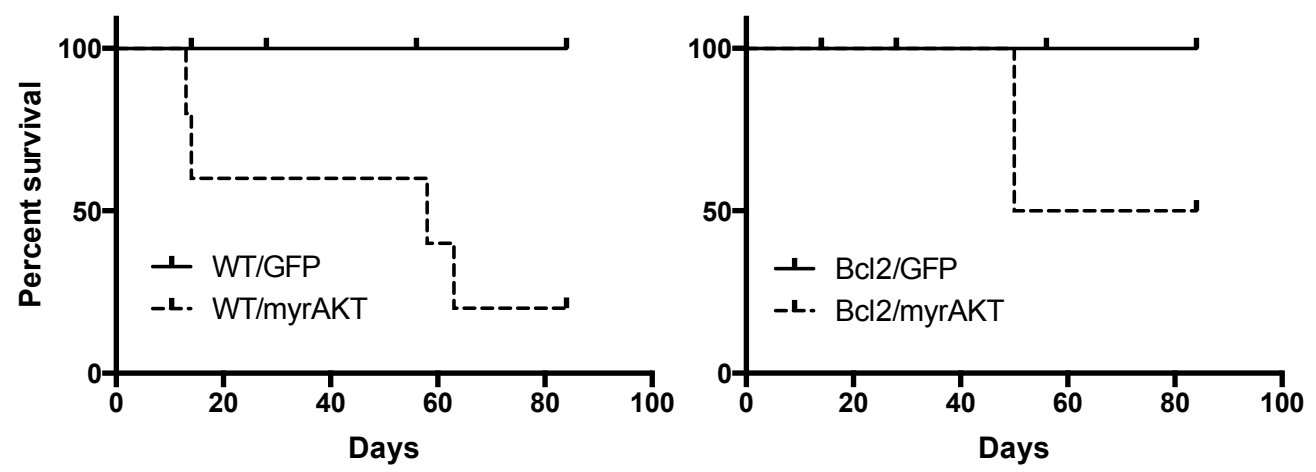

C

Intravenous Intrafemoral
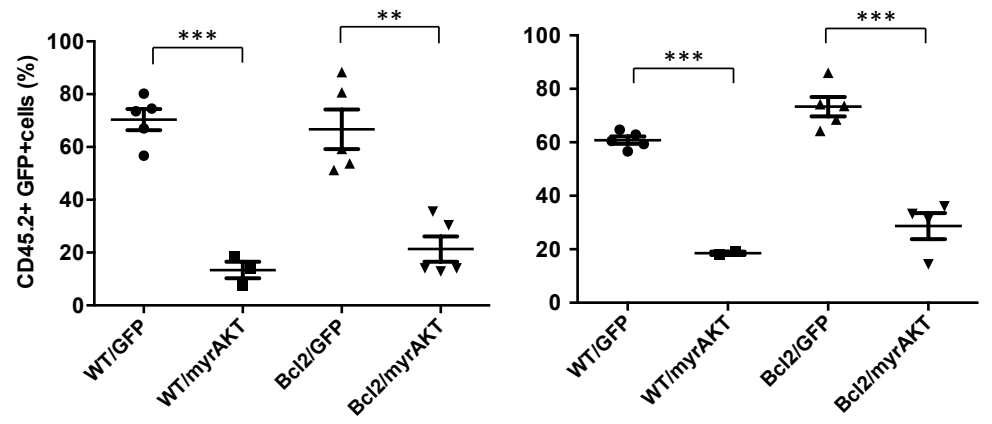

Figure 4 
a

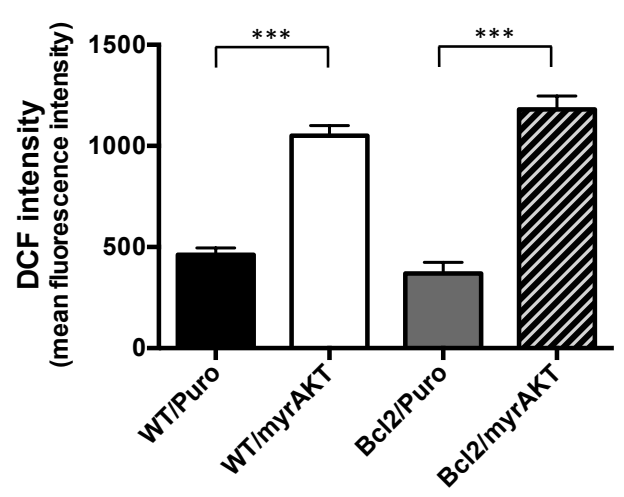

C

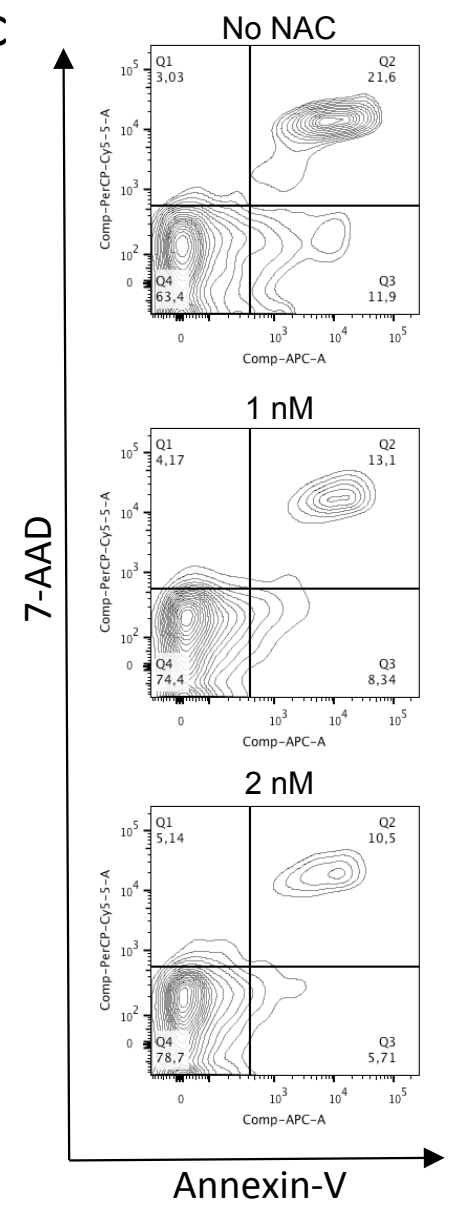

b

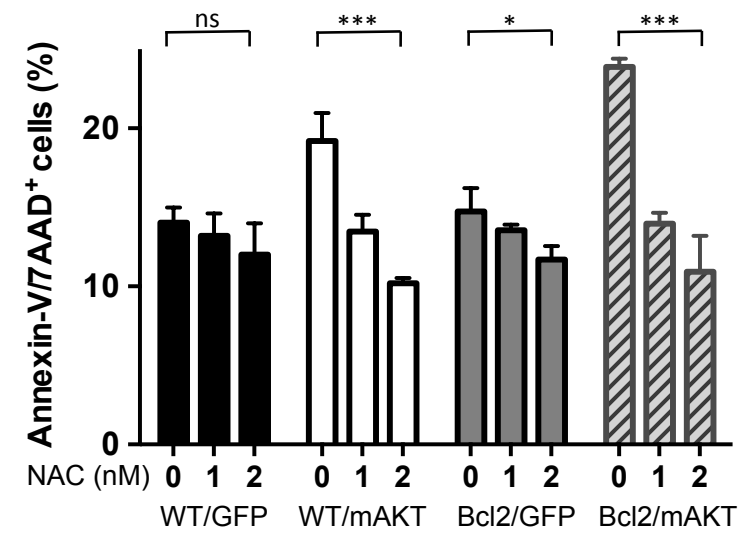

d

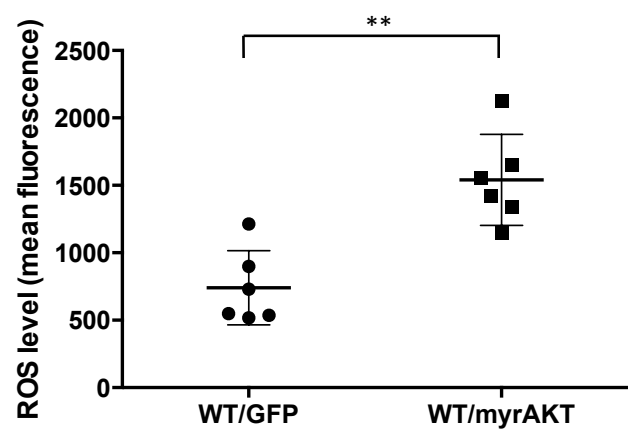

Figure 5 